\title{
Queer Studies SIG Members' Responses to the AERA Gender Identity Data Collection Strategy
}

May, 2016

A brief report prepared by:

Kamden K. Strunk, Ph.D.

Assistant Professor of Educational Research, Auburn University

Director, Research Initiative on Social Justice and Equity

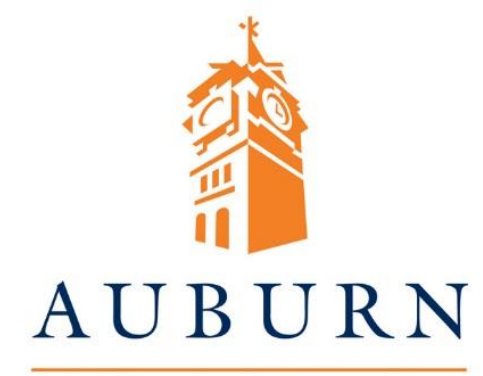

U N I V E R S I T Y

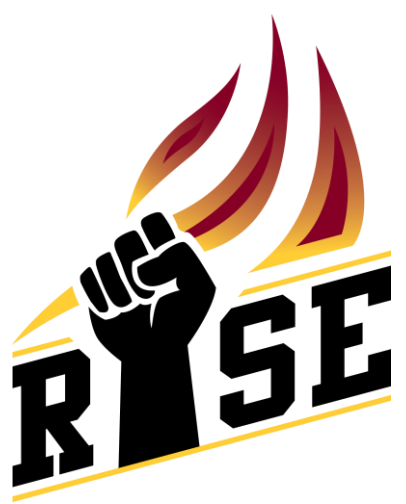




\section{Contents}

Executive Summary ...................................................................................................... 3

Queer Studies SIG Members' Responses to the AERA Gender Identity Data Collection Strategy4

History and Impetus for the Creation of the Current Survey ................................................. 4

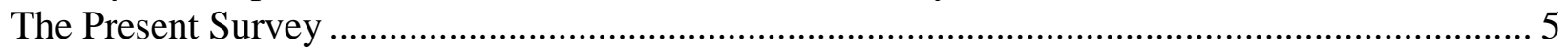

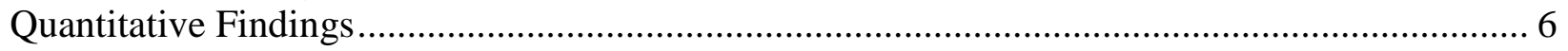

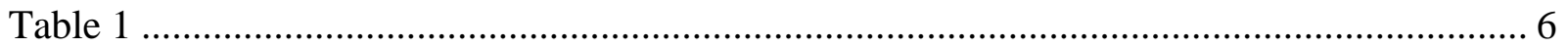

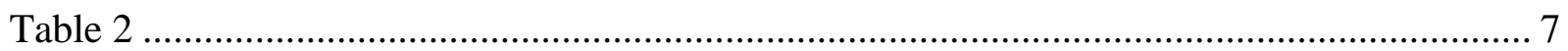

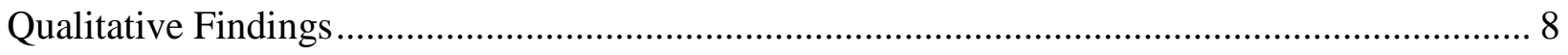

What are your thoughts on the options currently set to go into effect? ................................. 8

If you were to create a list of gender identity options for the AERA membership registration

form, which options would you include?...................................................................... 8

Please share any other thoughts you may have about the collection of gender identity

information as part of AERA's member registration process. ............................................ 9

Please feel free to share other comments related to this issue below ................................. 10

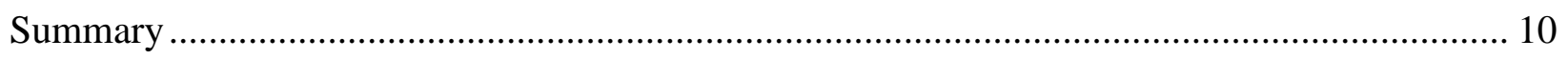

Appendix A: AERA Press Release on Gender Identity Data Collection.................................. 11

Appendix B: List of Gender Identity Options Suggested by SIG Members.............................. 13

Appendix C: Email Exchange via Unofficial SIG Listserv .................................................... 14

Appendix D: Email Exchange Between SIG Leadership and AERA Headquarters ................... 18 


\section{Executive Summary}

The present report presents data from a survey of the AERA Queer Studies SIG membership around newly-announced gender identity options for membership data collection. Both quantitative and qualitative data are presented that suggest many in the SIG membership are unhappy with current options, distrust the process by which they were created, and feel marginalized by the organization. However, the same data include suggestions for moving forward, constructive ideas for meeting data collection needs, and meaningful commentary on the current options and processes. Based on the present data, it seems that the newly announced options are unsatisfactory to most SIG members, but there is hope for constructive dialogue toward doing better, and creating an inclusive and equitable AERA.

NOTE: The writing and conclusions expressed in this report are the result of research by the individuals named on the title page of this report. Nothing in this report is intended to represent, nor should it be construed as representing, the position of Auburn University, or any other institution with which a RISE director, associate, affiliate, or fellow is affiliated. 


\section{Queer Studies SIG Members' Responses to the AERA Gender Identity Data Collection Strategy}

The purpose of this brief report is to summarize the responses of the Queer Studies SIG Membership to recent changes in how AERA collects gender identity information in the membership registration and renewal process. The Queer Studies SIG commissioned a membership-wide poll following the announcement of the new gender identity data collection strategy by AERA. The survey construction was approved by the SIG executive committee on May $4^{\text {th }}$, and data collection occurred from May $4^{\text {th }}$ through May $7^{\text {th }}, 2016$. This report gives a brief synopsis of the reasoning behind administering this survey, the quantitative results of the survey, and a snapshot of the qualitative results.

\section{History and Impetus for the Creation of the Current Survey}

On April 30 ${ }^{\text {th }}, 2016$, AERA issued a press release announcing an expansion of gender identity options in registration and renewal forms for membership. These options included:

- Female/Woman

- Male/Man

- Transgender Female/Transgender Woman

- Transgender Male/Transgender Man

- Another gender identity (please specify)

The same press release, which is attached to this report as an appendix, touted the new options, saying the "expanded categories go beyond the biological female and male identities to include transgender as well as man and woman, terms that tend to be defined by one's culture or society." The same press release stated that, "AERA developed these gender categories after exploring how peer research societies categorize gender and conducting a comprehensive literature review." The release went on to explain that AERA sought input from various constituencies, including the Queer Studies SIG, "as many of these scholars and researchers are well published and noted experts on gender issues."

Fairly immediately after the press release was issued, Queer Studies SIG members went to social media to express their feelings about the change. Within hours, there were two comment threads on the Queer Studies SIG Facebook page, including a combined total of 27 reactions and 34 comments. Among those initial comments were those expressing concern about the change, as well as a healthy number of individuals expressing frustration with the claim that Queer Studies SIG members were consulted.

Through the efforts of concerned SIG members, an email chain from May of 2015 has been discovered, which sheds some light on the current controversy. Initially, George Wimberly at AERA headquarters forwarded a message to the then-co-chairs of the SIG asking for input on a new set of gender identity options. This message was forwarded to the unofficial SIG listerv, which generated some discussion and additional questions. The full exchange may be found in Appendix C. It seems clear from that email chain that SIG members expressed hesitance about the proposed options, suggested many changes, and asked for clarification before moving forward. Based on the available evidence, it does not appear the SIG's questions were responded to by anyone at AERA headquarters. A full copy of the exchange between SIG leadership and 
AERA headquarters can be found in Appendix D, and their memo outlining proposed changes is in Appendix E. This email exchange makes clear that, even with the problems in SIG communication strategies at the time, the SIG leadership raised serious concerns about the proposed changes, and requested further information and consultation. It seems that AERA headquarters acknowledged the request for further consultation and information, but moved forward with changes anyway. Thus, while there appears to have been some level of consultation, neither the membership nor the leadership of the Queer Studies SIG appear to have endorsed any set of options. Instead, they appear to have merely asked questions in an attempt to create dialogue.

It is worth noting that the issue of multiple official and unofficial listservs, websites, and social media accounts has been an ongoing one for the SIG. It appears an unofficial listserv began as an attempt to create open lines of communication (the AERA official listserv does not easily accommodate open communication). However, many SIG members are not on the unofficial listserv, management of which is unclear. In sum, it seems there was some attempt at consultation, if not through the most accessible means, but that there was no consensus position among the membership, and that questions raised in that exchange went largely unanswered. This email exchange also illustrates the sense of marginalization many SIG members felt, even at that early stage.

The current report and survey are direct responses to that sense - that the voices of Queer Studies SIG members were not adequately included in the process. The primary goal of this report is to provide data and analysis related to a survey of the Queer Studies SIG membership, so that the SIG may collective provide meaningful input to ongoing conversations about gender identity within AERA.

\section{The Present Survey}

The present survey was administered via Qualtrics. It was sent via email to all active SIG members on May $4^{\text {th }}, 2016$, with a reminder email on May $6^{\text {th }}, 2016$. A total of 78 individuals responded to the survey, which is about $31.2 \%$ of the active SIG membership $(n=250)$. It is, perhaps, worth noting that of the 250 active SIG members, 117 opened the email invitation to participate. In other words, about $53.2 \%$ of SIG members never actually opened the email invitation to participate in the survey. This is perhaps due to issues such as spam filters (the email was sent from a Qualtrics email address, and thus might have been filtered out as bulk mail by some email systems), or the timing as the survey was sent at the end of the semester for many universities. These issues may present challenges to generalizability. However, the present report aims to provide a sense of the reaction of those who responded to the survey. These views are diverse, and represent at least some of the approaches to this issue taken by the Queer Studies SIG Membership.

The survey included four open-response items, and six Likert-type rating scales (all on a 7-point scale). The remainder of this report is dedicated to presenting and analyzing the results of that survey. First, quantitative data are presented to provide a quick sense of the mood of the membership, and then qualitative data are presented and interpreted thematically to provide deeper insights on the reactions and approaches of the membership. 


\section{Quantitative Findings}

The quantitative findings reveal a membership with diverse reactions to the current AERA change. For the present analysis, quantitative data are reported two ways: First, data were dichotomized into 'agree' and 'disagree' categories. This was accomplished by grouping responses including "Strongly Agree," "Agree," and "Somewhat Agree" into one "Agree" category, and by grouping "Strongly Disagree," "Disagree," and" Somewhat Disagree" into one "Disagree" category. "Neither agree nor disagree" responses were disregarded for this dichotomization. Second, the data are reported for all response categories on the 7-point Likerttype scale.

The dichotomized data are presented below:

\section{Table 1}

Responses to Likert-Type Options by Agree/Disagree Dichotomy

\begin{tabular}{lll}
\hline \multicolumn{1}{c}{ Item } & $\%$ Agree & $\%$ Disagree \\
\hline $\begin{array}{l}\text { I am satisfied with the newly announced list of gender identity } \\
\text { options for AERA membership purposes. }\end{array}$ & $11.4 \%$ & $86.1 \%$ \\
& & \\
$\begin{array}{l}\text { I understand the purpose for collecting gender identity information as } \\
\text { part of AERA membership registration }\end{array}$ & $43.0 \%$ & $48.1 \%$ \\
$\begin{array}{l}\text { I feel confident that AERA, as an organization, is striving for a more } \\
\text { inclusive and equitable membership experience. }\end{array}$ & $50.0 \%$ & $41.0 \%$ \\
$\begin{array}{l}\text { I feel confident that the AERA leadership has good intentions with } \\
\text { the changes to gender identity data collection. }\end{array}$ & $66.7 \%$ & $25.6 \%$ \\
$\begin{array}{l}\text { AERA has done a good job on issues of gender identity. } \\
\begin{array}{l}\text { I feel like my input is valued on issues of gender diversity by AERA } \\
\text { as an organization. }\end{array}\end{array}$ & $25.6 \%$ & $47.4 \%$ \\
\hline
\end{tabular}

These dichotomized data are illustrative of several ideas: The SIG membership is largely unhappy with the newly announced options. Almost half of the SIG membership does not understand the purpose for AERA collecting gender identity information. Only about half of the membership feels confident that AERA is working toward inclusivity and equity. About a quarter of the membership does not trust that the AERA leadership has good intentions around gender identity. Over two-thirds do not feel AERA has done a good job on gender identity issues. Finally, a little less than half the SIG membership feels their input is not valued by AERA. However, it seems that many in the SIG understand the new identity options as a goodfaith effort by AERA, even if the effort was not successful.

This same pattern of findings emerges in the data when they are viewed across all seven response categories (versus the dichotomized data presented above). These more comprehensive results are shown below. 
Table 2

Responses to Likert-Type Rating Scales

40

30

25

20

15

10

I am satisfied with the newly announced list of

gender identity options for

AERA membership

purposes

- Strongly agree

I understand the purpose for collecting gender ntity information as part AERA membersh

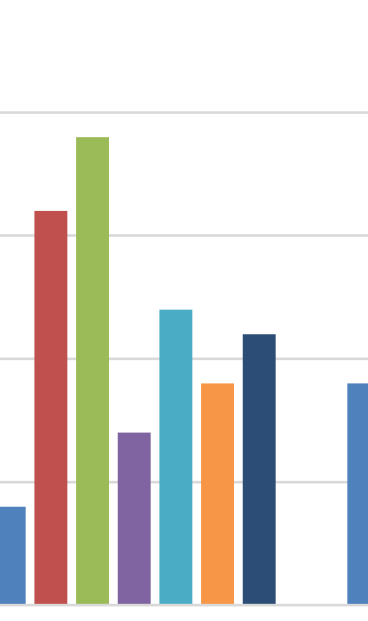

feel confident that AERA, as an organization, is striving for a more inclusive intentions with the changes and equitable membership to gender identity data experience. collection
AERA has done a good job I feel like my input is valued on issues of gender issues of gender diversity by AERA as an organization 


\section{Qualitative Findings}

In this section, the qualitative findings are presented and analyzed. For the sake of ease of presentation, the data are presented by the question to which Queer Studies SIG members were responding.

\section{What are your thoughts on the options currently set to go into effect?}

By far the most common response in the open-ended data to the newly announced gender identity options was that the new options "falsely conflate sex and gender". Dozens of members described the combination of categories like "male" and "man", "female" and "woman" as problematic. Another frequent comment was that these options "reinforce binaries" and fail to "[recognize] the spectrum of identities related to gender identity." Also prevalent in the responses were questions about the necessity or advisability of separating cisgender and transgender in a stark fashion. One response, potentially, captures some of what is behind the others: "AERA wants 'stable identity variables, which rejects MOST of the research on queer identities." The same respondent points out that queer notions of identity are "inherently unstable_-and dangerously NOT quantifiable."

A second prominent theme in responses to the newly announced gender identity options is the lack of a place for nonbinary individuals. Several respondents suggest the lack of options for nonbinary individuals leaves them othered. One SIG member pointedly asked, "Am I still 'other'?" Another suggests that leaving nonbinary individuals in the 'other' category suggests AERA views those individuals as "marginal and fringe."

A number of members in response to this item by questioning why AERA needs to know about trans/cis status at all. One respondent, for example, suggests that some members are unlikely to "out himself/herself" and might answer intentionally inaccurately or skip the item altogether. This comment ties to a deeper them running through many of the responses of distrust - members expressed not understanding the need for gender data collection at all, and wondering what would be done with the data. One respondent observed, "No one asks hair color as it is not pertinent (i.e., used to empower or discriminate."

The SIG membership was not universally unhappy with these options, however. While the responses critiquing the new options far outweighed those in favor, a substantial group seemed in favor of the new options. One called them "fair and inclusive," another "comprehensive". One participant even questioned if the move included too many different identities, saying the list "seems like a lot."

\section{If you were to create a list of gender identity options for the AERA membership registration form, which options would you include?}

When asked which options SIG members would include if it were up to them, many simply stated they would not create such a list at all. One respondent answered the question bluntly: "I would not." Other suggest such a list is not productive, and suggest a "qualitative response" or a "blank for people to write in how they identify." Many others suggested adding 
"queer" or "genderqueer" to the list. Several provided long lists intended to be more comprehensive, and a few others suggested other organizations as a resource, including The Williams Institute, and the Human Rights Campaign.

A number of members suggested such a list could not be constructed by any individual or team of individuals, and instead should involve conversation and polling of "the entire AERA membership". Still others suggested that their colleagues in the Queer Studies SIG would be better equipped to compile such a list, if one is necessary at all.

A list of options suggested by Queer Studies SIG members can be found in Appendix B. It is worth noting that many members declined to provide a list, citing the impossibility of adequately capturing the lives of individuals with any such list. A number of others suggested that it might be more productive to collect data on pronoun usage. Several suggested such pronoun usage data could be optionally printed on AERA conference badges, which could create a more welcoming environment as well as capture something about the composition of the membership.

\section{Please share any other thoughts you may have about the collection of gender identity information as part of AERA's member registration process.}

Responses to this item were varied. Some salient quotes are excerpted below:

- "I think AERA needs to include, on the page where this information is collected, a statement about what this data is being collected for and who has access to it."

- 'I don't know what the purpose is. Is it to ensure representation? Is it to rethink policies? All of the above? None of the above?"

- “AERA hasn't earned my trust in this item. I will most likely decline to state."

- "Collecting gendered information presumes it is a salient feature of the social world, but also makes sure it is, by having all information read through that tiny lens."

- "Unless the information is being used to identify potential patterns of discrimination, I don't see it as valuable."

- "I get why the information is helpful and I like that attempts were made to be more inclusive of gender identity but I think, to capture the reality of gender identity, efforts should be made to include gender diversity options with the assistance of scholars who study gender diversity."

- "The trouble of collecting gender identity is that it is often used under the guise of diversity or representation. However, these attempts usually result in marginalizing or reminding gender minorities of their minority status."

- "This will be my last year as a member of AERA. They don't value gender identity or fluidity. I asked several times in Chicago and DC where the gender neutral bathrooms were and no one could tell me. They don't value queer research. Their positivistic epistemological foundations don't recognize my body or my experience. Thus, they won't get my membership or money. After two years, I've had it."

- "Why is this collected? Is it helpful or meaningful? Perhaps it is, and I am happy if that is the case. However, gender information is often collected pro forma and purposelessly."

- "AERA should leave a space for people to self identify for 3 years, and use responses to help create categories for the following 5 years; then repeat the process" 
A number of members used this item to express their disappointment with not being consulted or allowed an opportunity to provide input prior to the press release. One suggested that "AERA should collect information from multiple stakeholders before making such changes - not just contacting representatives from particular groups or SIGS.” Another specifically suggested that the Queer Studies SIG Business Meeting would have been a productive space to have a conversation about these ideas.

\section{Please feel free to share other comments related to this issue below}

A large number of responses to this final, catch-all, question were related to AERA as an organization. Many commented on a perceived lack of trans-inclusive and gender-neutral restrooms at the convention. Many others expressed again their frustration at not feeling consulted or valued in the process of creating these new options.

One respondent dug through old emails to determine when/if there had been some level of consultation. That SIG member found a message from May, 2015 sent via the SIG listserv that sought input from AERA leadership. The respondent reported there was no real resolution in those messages, and that individuals has forwarded "questions" but "not a lot of consensus". That member suggests that "there are still many issues of concern in the ways they approach LGBTQ topics, but...at least a modicum of consultation did happen on this topic last year."

\section{Summary}

From the present data, a few key themes emerge: A dissatisfaction with the newly announced options; A more general dissatisfaction with attempts to quantify queer bodies; A sense of marginalization and exclusion in the process of creating these categories; A desire for a more deeply deliberative and consultative process for such decisions. It appears many SIG members feel marginalized within AERA (physically and in power dynamics), and have developed a level of distrust for AERA leadership around LGBTQ issues.

The present data also offer many options for moving forward. Many members who responded to the survey appeared willing to engage in productive dialogue on this issue, and offered suggestions for how to move forward. These include the use of open-ended gender identity responses, "check all that apply" gender identity options, a broader list of available gender identity options, or perhaps dropping gender identity data collection altogether. It is not the purpose of this report to suggest a solution - but Queer Studies SIG members presented many possibilities and suggested a willingness to engage in the process of meeting organization data collection needs while valuing the lived experiences of members. 


\title{
Appendix A: AERA Press Release on Gender Identity Data Collection
}

\author{
AERA Expands Gender Category Options for Members
}

\section{April 2016}

Beginning in 2017, AERA members will have the option to select from an expanded list of gender categories when renewing their membership or joining the Association. Recognizing that the traditional gender category options of "female," "male," and "other gender" fail to capture the full spectrum of gender identities and expressions, AERA Council approved the use of a new gender identity question and an expanded set of gender categories at its February 2016 meeting. AERA's demographic data collection will now include five gender categories, as follows:

Which best describes your gender identity?

Female/Woman

Male/Man

Transgender Female/Transgender Woman

Transgender Male/Transgender Man

Another gender identity (please specify):

This updated question and expanded categories go beyond the biological female and male identities to include transgender as well as man and woman, terms that tend to be defined by one's culture or society. Members also have the option of selecting "another gender identity" and specifying their gender. This charge will enable AERA to examine how individuals identify themselves and allow the Association to craft response options in the future to capture these expanded gender identities. Responding to the gender identity question on membership forms remains optional.

"In addition to using gender demographics for membership and research purposes, the expanded and enhanced gender categories send a message of inclusion to individuals of all gender identities within the Association,” said AERA Executive Director Felice J. Levine.

AERA developed these gender categories after exploring how peer research societies categorize gender and conducting a comprehensive literature review. AERA staff solicited guidance and input from members of the Committee on Scholars \& Advocates for Gender Equity in Education (SAGE), the Social Justice Action Committee, and the Queer Studies SIG, as many of these scholars and researchers are well published and noted experts on gender issues. Staff also engaged in discussions with federal agencies to learn to what extent and how the collection of gender data is being rethought in major federal data collections or funded studies.

The changes implemented to the gender categories are part of a larger effort begun in 2013 to update and improve the data AERA collects about the demographic characteristics of its members. In addition to data on gender identity, AERA collects other member demographics such as race/ethnicity, degrees earned, and age in order to understand and describe the composition of its membership. A similar expansion of the race/ethnicity categories was recently implemented to reflect the diverse and multiple racial and ethnic groups AERA members 
represent. These expanded categories enable AERA to examine and report on member demographics overall, as well as by Division, SIG, and Committee. Member demographics are reported to AERA Council and the Affirmative Action representatives in each Division to examine overall trends in AERA's membership and participation in Association activities such as the Annual Meeting.

In some cases, membership data are used for research purposes, such as efforts to understand the field and profession of education research. Regardless of how the data are being used, AERA holds to a high standard of data security and research ethics. Individual member information is kept confidential and only reported in aggregate form. AERA does not report gender identities or other demographic information where there are a small number of cases that might inadvertently identify specific members.

Retrieved from: http://www.aera.net/Newsroom/AERAHighlightsE-newsletter/ AERAHighlights-April2016/AERAExpandsGenderCategoryOptionsforMembers/ tabid/16298/Default.aspx 


\section{Appendix B: List of Gender Identity Options Suggested by SIG Members}

NOTE: This list is provided in alphabetical order. It is also important to note that most SIG members declined to provide a list, suggesting instead that open-ended responses would be better. Many also suggested that instead of capturing gender identity, AERA would be better served by capturing pronoun usage. This list is perhaps best in illustrating the huge range of labels with which members identify, thus making reducing those to a manageable list for quantitative data collection difficult or impossible.

Agender

Agenderflux

All-gender

Androgyne

Androgynous

Aporagender

Assigned Female at Birth

Assigned Male at Birth

Bigender

Butch

Cisgender

Cisgender Man

Cisgender Woman

Demiboy

Demigender

Demigirl

Enby

Female identifying

Feminine Identified

Femme

FTX

Gender fluid

Gender Neutral

Gender Non-Conforming

Genderless

Genderqueer
Intersex

Male identifying

Man

Masculine Identified

Maverique

MTM

MTN

MTX

Neutrois

Nonbinary

Pangender

Queer

Trans Man

Trans Woman

Trans*

Transfeminine

Transgender

Transgender Man

Transgender Woman

Transmasculine

Two-spirit

Vaguegender

Woman

X-Gender

XTX 


\section{Appendix C: Email Exchange via Unofficial SIG Listserv}

The below email exchange took place in May, 2015, in response to a request from AERA headquarters to the Queer SIG for input on changes to gender identity options. All contact information has been redacted for the purposes of this report, except for AERA headquarters employees, to provide those reading this report with appropriate context without divulging identifying information for participants in this exchange.

From: AERA Queer Studies Special Interest Group <AERA-QUEER-STUDIES-

SIG@LISTSERV.UA.EDU> on behalf of [REDACTED]

Date: Tuesday, May 12, 2015 at 7:49 AM

To: "AERA-QUEER-STUDIES-SIG@LISTSERV.UA.EDU" <AERA-QUEER-STUDIESSIG@LISTSERV.UA.EDU>

Subject: Re: AERA Gender Memo and Queer Studies SIG

Thank you all for these wonderful posts and conversations. I attempted to reflect this in my response to George. The two biggest concerns I raised for him were (1) we are yet again only brought in as an afterthought and then given the robustness of our exchange, we are clearly intellectual leaders in this area and (2) this is a difficult conversation without fully understand the intent. I copied and pasted the wonderful replies here for him. I am finished my term as chair but hope there can remain some energy around this issue. While we ay never have the ear of AERA, I know that many institutions are wrestling with this same decision to be more inclusive and collect more data about sex and gender identity. Threads/discussions like this one are good for our communities.

Stay well,

[REDACTED]

On Thu, May 7, 2015 at 3:56 PM, [REDACTED] wrote:

My first question was why do they need to collect that information to begin with, i.e. what assumptions are they making about gender? Then I realized we should suggest they replace the male and female categories with cisgender. I wonder how George will react to that?

\section{[REDACTED]}

From: AERA Queer Studies Special Interest Group [mailto:AERA-QUEER-STUDIES-

SIG@LISTSERV.UA.EDU] On Behalf [REDACTED]

Sent: Thursday, May 7, 2015 3:02 PM

To: AERA-QUEER-STUDIES-SIG@LISTSERV.UA.EDU

Subject: Re: AERA Gender Memo and Queer Studies SIG 


\section{Hi [REDACTED],}

I'm curious about the perception of trans* as transmisogynistic--I had never heard that perspective before. Can you explain further? I'm happy to hear from you off-list, to keep people's inboxes unclogged.

\section{[REDACTED]}

On May 7, 2015, at 1:46 PM, [REDACTED] wrote:

This goes to show how problematic these terms are. I myself identify as trans and advise a university student group of transgender and non-binary students and sit on the board of directors of a leading trans advocacy organization, and it is from within my lived experience that I speak. Trans women in the groups with which I work are opposed to the term trans*. Agender persons within the communities where I work feel marginalized when an -ed is added to the end of agender... Just as we in the transgender community do. No experience is universal. All research needs to be problematized and left room to grow and to learn from the experiences of others. Trying to put 7 billion people in 4 boxes will always cause strife. We should ask each individual what they want to identify as rather than trapping them in categories we assume are correct.

On May 7, 2015 12:26 PM, [REDACTED] wrote:

[REDACTED]. I don't know you but I disagree about the trans* and agender comment-- in fact I have 4 pubs and 2 books coming out that highlight those terms.

I'm happy to discuss this further. I live these identities and have deep research within my communities about them.

[REDACTED]

Sent from my iPhone

On May 7, 2015, at 11:06 AM, [REDACTED] wrote:

Trans* is often seen as trans misogynistic, and agendered has that problematic -ed ending, plus by selecting a few categories to list, you are leaving out many others. This is a complicated conversation that we should have been included on from the beginning and there is no easy answer.

On May 7, 2015 11:48 AM, [REDACTED] wrote:

How about FTM, MTF, true gender, gender queer, trans*, agendered, gender creative

[REDACTED]

On May 7, 2015, at 10:37 AM, [REDACTED] wrote: 
I want to second Tim's point, having just come of the AERA handbook on GLB, no, thanks not right, LGB, no, not quite, LGBTQ issues in education *SNARK!*.

There is a bias against the queer studies sig by AERA central, to some extent, because of our name--QUEER. I'm quite serious on this point. Madelaine and I got some pressure on our chapter to rename our theoretical framework--QUeer Legal Theory, to some thing else. I had to point out that QLT has been around since 1995, per Francisco Valdes.

\section{*SIGH!*}

From my perspective, the very last minute inclusion of the Queer Studies SIG is because SAGE pushed George to contact us. This is speculation on my part, but it's based on a long, unhappy of being treated like uppity hired help.

Just sayin.

[REDACTED]

From: [REDACTED]

To: AERA-QUEER-STUDIES-SIG@LISTSERV.UA.EDU

Sent: Thursday, May 7, 2015 12:07 PM

Subject: Re: AERA Gender Memo and Queer Studies SIG

[REDACTED],

It is good that George is requesting our input, put I can't help feeling slighted when the Queer SIG was not even considered for consultation in their original proposal. It was not until another SIG suggested that he contact us that he did so. The impression I get is that he was essentially forced to reach out to us. All of which makes me wonder how our suggestions and ideas will be received and considered.

Apologies for the downer on the finest spring day of the year so far (in Michigan!)

[REDACTED]

Sent from my iPhone

On May 6, 2015, at 2:59 PM, [REDACTED] wrote:

Greetings all,

I received this message from George Wemberly related to changes in gender categories used for membership. I am glad they are asking for our feedback and am sure many of you have thoughts about this. If you reply to me directly by May 11, I can pull together some general themes raised to forward. You can also reply to George. His email is in the message.

Stay well,

[REDACTED] 
From: George L. Wimberly <gwimberly@aera.net>

Date: Wed, May 6, 2015 at 1:33 PM

Subject: AERA Gender Memo and Queer Studies SIG

To: [REDACTED]

Cc: Nathan Bell <nbell@ aera.net>

Hello [REDACTED],

I hope this message finds you both well. I want to share with you and the Queer Studies SIG a memo AERA Central Office drafted regarding updating the gender categories we use for membership. We initially sent this memo to the Committee on Scholars for Gender Equity (SAGE) and the Social Justice Action Committee for their insight and suggestions. This topic was first discussed last fall and we followed-up at the 2015 Annual Meeting. SAGE members encouraged us to share this memo with the Queer Studies SIG for further input.

As discussed in the memo, we are considering expanding possibly expanding our membership gender categories beyond "female, male, and other". We outline a couple of options in the memo. We plan to make a recommendation to AERA Council this June regarding the gender categories.

We welcome any suggests or guidance members of the Queer SIG can give us. We would prefer any suggestions no later than June 1st to allow us to prepare for the next AERA Council Meeting.

We value your expertise and would welcome either a conversation (conference call) or written suggestions. Thank you in advance for your consideration. Feel free to contact me with any questions or concerns.

Regards,

George

George L. Wimberly, Ph.D.

Director of Professional Development

Diversity Officer

American Educational Research Association (AERA)

1430 K Street NW, Suite 1200

Washington, DC 20005

Office Phone: (202) 238-3225

Cell Phone: (301) 437-9145

gwimberly@aera.net

<AERA DemographicsGenderData_BriefingMemo_4-18-15.pdf> 


\section{Appendix D: Email Exchange Between SIG Leadership and AERA Headquarters}

The below email exchange took place in May, 2015, and is between SIG leaders (whose names and information have been redacted) and AERA headquarters employees.

From: "George L. Wimberly" <gwimberly@aera.net>

To: [REDACTED]

Cc: [REDACTED], "Nathan Bell" <nbell@aera.net>

Sent: Tuesday, May 12, 2015 10:44:12 AM

Subject: RE: AERA Gender Memo and Queer Studies SIG

Hello [REDACTED],

Thanks for sharing the memo with the Queer Studies SIG members and soliciting their feedback. As you see, there are many informed suggestions and opinions on this issue. We will make a recommendation to Council in the coming weeks; however, we will likely need to re-visit the issue in the future as we have further research and societal definitions of gender evolve.

We will keep you and the Queer Studies SIG members posted on our recommendation and the ultimate decision. Thank you again.

Regards,

George

From: [REDACTED]

Sent: Tuesday, May 12, 2015 9:28 AM

To: George L. Wimberly

Cc: [REDACTED]; Nathan Bell

Subject: Re: AERA Gender Memo and Queer Studies SIG

George,

Thank you for reaching out to the Queer SIG for thoughts about this. We love to be involved in these conversations. Our only hope is that in the future as this is revisited, we will be invited into the conversation earlier.

I asked for and received lots of wonderful feedback from members and asked people to also reach out to you directly. There is clearly not consensus and the great conversation we had on the listserv is a good indication that a public meeting about this in the future will be healthy and educative for AERA. I have posted posts below. One theme really relates to distinguishing between and being clear about whether you are asking about sex or gender. And you may need to ask about sex and gender identity to really capture what you want. A second theme is the 
purpose of the question. Is this to collect data or to show an openness to all communities and persons.

I hope this is helpful.

\section{[REDACTED]}

1. There needs to be an option in all categories for intersex

2. I'm so glad this issue is being addressed! Putting my two cents in, I would strongly prefer Option B listed in the memo, with a few changes. Personally I think of 'male' and 'female' as labels of biological sex, not gender. So, are they really asking for sex or gender? As a non-binary trans* person who isn't transitioning, I wouldn't exactly know what to answer on Option B; I am female, but that's not my gender; I am more than gender non-conforming, I am trans*; but, I am not a transgender man. So my personal suggestion would be to eliminate 'male' and 'female' completely and replace them with 'man' and 'woman'. I believe we need to be clear about the difference between sex and gender/gender identity; stop blurring those lines and recognize that one's male or female parts does not determine their gender.

I'm also unsure about combining genderqueer and gender non-conforming; as it is written, how would a gender non-conforming/butch woman answer? In my understanding and experience, I haven't found gender non-conforming to be a gender identity in itself; as I understand it, 'gender non-conforming' is generally used to describe a cisgender person whose gender expression may be transgressive in some way, but that person still identifies as a cisgender man or cisgender woman. But, perhaps others use this label differently. If my interpretation is generally accurate then I might suggest removing 'gender non-conforming' and replacing it with with 'trans*' with an asterisk.

3. I wanted to put in my two cents in favour of Option A (male, female or other as selfspecified in writing). As a gender scholar and genderqueer-identified person, I think that the openness is both equitable and scholarly as it invites further data from members on how we might use the categories instead of implanting a schema. Furthermore, there is a question of what the data is for and how it is used. Many, many people with a trans history of some kind identify as men or women without referencing that history, and many more might identify openly with that history in some areas of their lives and not professionally. Or, they might have a trans history or identification but for political or other reasons identify themselves as (cis) male or female. So, it begs the question as to whether having male, female, transgender male and transgender female actually collects meaningful data at all (for whatever purpose). If the intent is simply to demonstrate inclusion (which I read in George's report and proposal) then I don't see why the question can't be left open. Lastly, trans people have a dizzying array of terms we use to refer to ourselves and each other, which will surely pop up on the list serve in the next few days as people make their suggestions toward Option B. Another point toward Option A, in my opinion. Please pass this onto George, if it would be useful to do so. 
4. Is there anyway to talk them out of using sex categories when they ask for gender? Also is there a reason they are lumping genderqueer and gender non-conforming together?

I would propose the following:

What is your sex or gender?

Male/man

Female/woman

Trans* (please describe)

Genderqueer

Gender non conforming

Agender

Another identity (please describe)

Make sense?

5. How about FTM, MTF, true gender, gender queer, trans*, agendered, gender creative

6. In my work, I refer to trans* a prefix or adjective used as an abbreviation of transgender, derived from the Greek word meaning "across from" or "on the other side of." Many consider trans* to be an inclusive and useful umbrella term. Trans (without the asterisk) is most often applied to trans men and trans women, and the asterisk is used more broadly to refer to all noncisgender gender identities, such as agender, cross-dresser, bigender, genderfluid, gender**k, genderless, genderqueer, non-binary, non-gendered, third gender, trans man, trans woman, transgender, transsexual and two-spirit.

On Wed, May 6, 2015 at 1:33 PM, George L. Wimberly <gwimberly@aera.net<mailto:gwimberly@aera.net>>wrote: Hello [REDACTED],

I hope this message finds you both well. I want to share with you and the Queer Studies SIG a memo AERA Central Office drafted regarding updating the gender categories we use for membership. We initially sent this memo to the Committee on Scholars for Gender Equity (SAGE) and the Social Justice Action Committee for their insight and suggestions. This topic was first discussed last fall and we followed-up at the 2015 Annual Meeting. SAGE members encouraged us to share this memo with the Queer Studies SIG for further input.

As discussed in the memo, we are considering expanding possibly expanding our membership gender categories beyond "female, male, and other". We outline a couple of options in the memo. We plan to make a recommendation to AERA Council this June regarding the gender categories. 
We welcome any suggests or guidance members of the Queer SIG can give us. We would prefer any suggestions no later than June 1st to allow us to prepare for the next AERA Council Meeting.

We value your expertise and would welcome either a conversation (conference call) or written suggestions. Thank you in advance for your consideration. Feel free to contact me with any questions or concerns.

Regards,

George

George L. Wimberly, Ph.D.

Director of Professional Development

Diversity Officer

American Educational Research Association (AERA)

1430 K Street NW, Suite 1200

Washington, DC 20005

Office Phone: (202) 238-3225<tel:\%28202\%29\%20238-3225>

Cell Phone: (301) 437-9145<tel:\%28301\%29\%20437-9145>

gwimberly@aera.net<mailto:gwimberly@aera.net> 


\section{Appendix E: AERA Memo on Gender Identity Options from April, 2015}

On the following pages is the memo that was received by SIG leadership in May, 2015, and forwarded via the unofficial SIG listserv. This memo may be illustrative of the options originally proposed, and how the SIG leaders at the time responded to those options. 
AERA Membership Data on Gender Demographics-Recommendations for Data Collection

\author{
Nathan E. Bell, Associate Director, Education Research and Research Policy \\ George L. Wimberly, Director of Professional Development; Diversity Officer
}

April 2015

Until recently, AERA membership demographic information, like most membership associations, national surveys, and data collection efforts, only used "female" and "male" gender categories in its collection of demographic data. Recognizing that these two categories fail to encompass all gender identities, AERA added "other gender" as a third response option in 2013 as part of a larger effort to update and improve upon the data that we collect about the demographic characteristics of our members. ${ }^{1}$ The addition of this third category is a step in the right direction, but there may be more effective or appropriate methods for capturing gender identity, especially in a diverse research society like AERA that values multiple gender identities and expressions.

Over the past few months, AERA staff (as a joint effort of our research program and diversity efforts) reviewed literature on gender categories and explored how our peer research societies categorize gender. We also engaged in discussions with federal agencies to learn to what extent and how the collection of gender data is being rethought in major federal data collections or funded studies. We plan to recommend to AERA Council in June 2015 how to categorize gender identity among AERA members as part of our demographic data collection.

\title{
Preliminary Findings
}

Our research has found that very few membership associations include gender categories beyond "male" and "female" in their demographic data collection. The American Psychological Association includes transgender as a category, and the American Society of Association Executives also includes transgender as a category, in addition to "prefer not to disclose." The association with the most comprehensive list of gender categories is the American Sociological Association (ASA), which began collecting data on the following categories in 2014:

- Male

- Female

- Transgender Male/Transgender Man

- Transgender Female/Transgender Woman

\footnotetext{
${ }^{1}$ The changes implemented in 2013 (for the 2014 membership year) also included updates to the race/ethnicity categories and other demographic questions. The race/ethnicity categories were refined and expanded, and members may now select multiple race/ethnicity categories. Throughout 2014, AERA proactively encouraged members to update their demographic data using these expanded demographic categories.
} 
- Genderqueer/Gender Non-conforming

- Preferred identity (in addition to or not listed above)

- Prefer not to say.

We did not identify any other membership associations that collected data on anything other than "female" and "male."

A small number of large-scale studies are capturing additional gender identities of their research subjects. The Youth Risk Behavior Survey, the National Survey of Student Engagement, and the National School Climate Survey, for example, each collect data using expanded gender categories. For the National School Climate Survey, individuals are asked to check all the following terms that apply to them: ${ }^{2}$

- Male

- Female

- Transgender

- Transgender Male-to-Female

- Transgender Female-to-Male

- If none of these terms apply to you, please tell us how you describe your gender

The National Survey of Student Engagement asks, "Are you transgender?" with response options of "yes" and "no.".

In most other federal data collection efforts, including all of the surveys conducted by the National Science Foundation, respondents are simply asked whether they are male or female.

While we have found some studies and membership associations that use expanded gender categories, there is little evidence that tests the validity and reliability of using these categories. A 2014 report from the Williams Institute provides the most explicit advice about how to ask questions about gender. ${ }^{4}$ This report is the culmination of an effort that started in 2011 in which the Williams Institute at the UCLA School of Law convened experts to "[map] the landscape of current practices to identify transgender and other gender minority respondents in population research, [assess] challenges to collecting data on gender-related aspects of individual identity, and [develop] strategies for establishing consistent, scientifically rigorous procedures for gathering information relevant to the needs and experiences of transgender people and other gender minorities." (p.i)

\footnotetext{
${ }^{2}$ Source: National School Climate Survey, 2011

${ }^{3}$ Source: National Survey of Student Engagement, 2013

${ }^{4}$ Best Practices for Asking Questions to Identify Transgender and Other Gender Minority Respondents on Population-Based Surveys, 2014, http://williamsinstitute.law.ucla.edu/wp-content/uploads/geniuss-report-sep2014.pdf.
} 
The Williams report recommends a two-step approach. Individuals are first asked what sex they were assigned at birth on their original birth certificate (male or female) and then are asked how they describe themselves (male, female, or transgender, with a fourth option for individuals who do not identify as female, male, or transgender). The Williams Institute is currently investigating whether the questions should be asked in the order presented or in reverse order, and is also researching a promising alternative approach to the second question on current gender identity, in which the response options are male, female, trans male/trans man, trans female/trans woman, genderqueer/gender non-conforming, and different identity (please state).

\section{Recommendations}

In expanding our gender categories, we also seek to signify to our members that we value gender diversity, while simultaneously respecting our members' right to privacy. However, collecting data on expanded gender categories is relatively new, we have few examples of ways in which we could collect such data, and the research on how best to collect the data is still ongoing.

We have arrived at two options. Option A proposes a minor change. We currently provide members with an "other gender" response option, but we do not ask them to specify their gender identity. With Option A, we propose adding a write-in box after the "other gender" category. This would enable us to see how individuals identify themselves and would allow us to craft response options in the future that capture these gender identities. Responding to the question would be optional as it currently is. Thus, with Option A, the AERA gender categories would be:

- Female

- Male

- Other (please specify):

With Option B, AERA would adopt an expanded list of gender options, similar to ASA and to the two-step process recommended in the Williams report. Again, responding to the question would be optional. Thus, with Option B, the AERA gender categories would be:

- Female

- Male

- Transgender male/transgender man

- Transgender female/transgender woman

- Genderqueer/gender non-conforming

- Different identity (please specify): 
We are now seeking additional input from the Committee on Scholars \& Advocates for Gender Equity in Education (SAGE) and the Social Justice Action Committee about these two options, given the expertise of these committees and their focus on gender and diversity issues. The Committees are not being asked to make a final recommendation, but rather are being asked to respond to these options and to provide additional suggestions about how demographics data collection on gender identity could be conducted by AERA.

With Option A, we would take a more cautious approach, allowing AERA to begin to collect data on expanded gender categories, while other organizations and individuals specializing in this area refine, test, and validate how questions on gender demographics should be asked. With Option B, we would use current (yet still evolving) best practices on how to collect data on gender identity, and test these best practices in our member demographics data collection efforts. With this option AERA would be one of a small number of membership associations attempting to collect such data in a consistent, structured way.

Regardless of the decision we make now, we recommend that AERA revisit this issue in two to five years, presumably after research in this area advances.

\section{Next Steps}

Based on input and suggestions from the Committee on Scholars \& Advocates for Gender Equity in Education (SAGE), and the Social Justice Action Committee (SJAC), and our ongoing research, we will prepare a final recommendation for the AERA Council, for consideration at their June 2015 meeting. We also plan to issue a briefing document that, we hope, will contribute to how other institutions, agencies, and research studies collect, analyze, and report on gender data in their work. 